\title{
Uso de las redes sociales en la promoción turística
}

\section{Use of social networks in tourism promotion}

1 Johanna Gabriela Monge Martínez https://orcid.org/0000-0001-6045-2410

Universidad Técnica de Ambato. Carrera de Turismo. Tungurahua.

jg.monge@uta.edu.ec

2 Gloria Estefanía Monge Martínez

Corvinus University of Budapest. Communication and Media Studies. Budapest.

martinez.monge@stud.uni-corvinus.hu

3 Carmen Isabel Vaca Vaca iD https://orcid.org/0000-0001-5576-0160 Universidad Técnica de Ambato. Carrera de Turismo. Tungurahua.

carmenivacav@uta.edu.ec

4 Fernando Israel Guerrero Ochoa

Universidad Técnica de Ambato. Carrera de Turismo. Tungurahua.

fguerrero7373@uta.edu.ec.

Artículo de Investigación Científica y Tecnológica

Enviado: 24/12/2021

Revisado: 29/12/2021

Aceptado: 12/01/2022

Publicado:08/03/2023

DOI: https://doi.org/10.33262/concienciadigital.v6i1.4.2002

Cítese: $\quad$ Monge Martínez, J. G., Monge Martínez, G. E., Vaca Vaca, C. I., \& Guerrero Ochoa, F. I. (2023). Uso de las redes sociales en la promoción turística . ConcienciaDigital, 6(1.4), 350-368. https://doi.org/10.33262/concienciadigital.v6i1.4.2002

CONCIENCIA DIGITAL, es una Revista Multidisciplinar, Trimestral, que se publicará en soporte electrónico tiene como misión contribuir a la formación de profesionales competentes con visión humanística y crítica que sean capaces de exponer sus resultados investigativos y científicos en la misma medida que se promueva mediante su intervención cambios positivos en la sociedad. https://concienciadigital.org

La revista es editada por la Editorial Ciencia Digital (Editorial de prestigio registrada en la Cámara Ecuatoriana de Libro con No de Afiliación 663) www.celibro.org.ec 
Palabras

claves:

redes sociales, promoción turística, marketing turístico

Keywords:

social

networks, tourism promotion, tourism marketing

\section{Resumen}

Introducción. Es relevante discutir que la promoción turística se puede realizar tanto en el entorno físico como virtual, donde es posible llegar a mayor audiencia trascendiendo barreras físicas como la distancia. Debido al confinamiento y el cambio de modalidad de aprendizaje de los presencial a lo virtual se utilizó la herramienta tecnológica virtual Instagram para dar a conocer los sitios turísticos del Ecuador. Objetivo. La presente investigación pretende describir cómo el uso de las redes sociales permite la promoción turística de los destinos. El contenido publicado en esta red fue desarrollado por los estudiantes como suplemento voluntario donde los alumnos podían compartir información turística. Metodología. Para analizar el impacto del contenido turístico generado en la plataforma se aplicó el modelo PRGS propuesto por IAB Spain Research que se fundamenta en las 4 variables presencia, respuesta, generación y sugerencia, las tres últimas unidas en una sola variable denominada engagement. Resultados. Existen 289 publicaciones turística. El $77 \%$ son turistas nacionales y el 23\% internacionales. El alcance es 3702 usuarios. Conclusión. En consecuencia, se pudo entender que las redes sociales tienen el potencial de ser un espacio de práctica complementaria para el estudiante y a su vez como un espacio de promoción turística.

\section{Abstract}

Introduction. It is relevant to discuss that tourism promotion can be carried out both in the physical and virtual environment, where it is possible to reach a larger audience by transcending physical barriers such as distance. Due to confinement and the change in learning modality from face-to-face to virtual, the virtual technological tool Instagram was used to publicize the tourist sites of Ecuador. Target. This research aims to describe how the use of social networks allows the tourist promotion of destinations. The content published on this network was developed by students as a voluntary supplement where students could share tourist information. Methodology. To analyze the impact of the tourist content generated on the platform, the PRGS model proposed by IAB Spain Research was applied, which is based on the 4 variables presence, response, generation and suggestion, the last three united in a single variable called engagement. Results. There are 289 tourist 
publications. $77 \%$ are national tourists and $23 \%$ international. The scope is 3702 users. Conclusion. Consequently, it was possible to understand that social networks have the potential to be a space for complementary practice for the student and, in turn, as a space for tourism promotion.

\section{Introducción}

Es en el año 2004 cuando se empieza hablar de la web 2.0, también denominada web social. Se trata de aplicaciones que generan colaboración y servicios (Fernández, 2014). Brinda a los centros educativos la posibilidad de crear espacios de encuentro e intercambio entre los docentes y los alumnos (Abuín, 2009). Facebook (FB) es un ejemplo de red social 2.0. (Cerdà y Planas, 2011). A su vez Instagram es una aplicación que facilita la construcción participada entre usuarios y marcas, debido, al elevado nivel de interacción (Caerols, et al., 2013). El número de fotografías que almacena la aplicación da a su vez prueba del elevado nivel de interacción de los usuarios con las marcas que participan en la aplicación. La importancia y la repercusión que han adquirido las redes sociales en los últimos años son indiscutibles. Instagram es uno de los medios que más ha crecido en los últimos años se trata del "mayor caso de éxito de la última década en el universo de las redes sociales" (Caerols, 2018, p. 229). Instagram es una aplicación de procedencia estadunidense, propiedad de Facebook ahora Meta, cuya funcionalidad primordial es poder compartir fotografías y vídeos con otros usuarios al instante e interactuar con ellos por medio de hashtags, comentarios, mensajes privados o me gustas (Figuereo-Benítez et al., 2021). Instagram tiene la cuarta mayor cantidad de usuarios de todas las aplicaciones móviles superado solo por Facebook, WhatsApp y Facebook Messenger todos de la misma empresa Meta. Más de mil millones de personas utilizan Instagram cada mes, de los cuales el $81 \%$ lo realizan para hallar un producto o servicio y de dichos el 50\% de los individuos ha visitado un portal web para hacer una compra luego de ver un producto o servicio en la plataforma (Newberry, 2021).

Facebook e Instagram son un gran potencial para la educación, a pesar de no haber sido concebidas como un entorno para construir y gestionar experiencias de aprendizaje. 
Funcionan como plataformas abierta, a diferencia de otros sistemas organizados en torno a cursos o contenidos formalmente estructurados (Cerdà y Planas, 2011). Aunque todavía siguen siendo algo poco presente en el ámbito académico, las ocasiones en que se utilizan son aisladas y pocas gozan de continuidad (Espuny et al., 2011). Las redes sociales son herramientas útiles para que un grupo de personas puedan potenciar su comunicación, sentirse parte de una comunidad y cooperar entre ellas en tareas comunes. Estas características pueden ser favorables para los entornos educativos. Estos espacios se pueden utilizar con el fin de potenciar la participación del alumnado en el proceso de aprendizaje, la interacción y motivación, la creatividad y la creación de redes de colaboración e intercambio (Garrigós et al., 2010). Los docentes deben adaptarse a esos cambios para que la escuela no quede anticuada y no continúe con el problema de los profesores quienes en el siglo XX intentan educar a jóvenes del siglo XXI en unas escuelas del siglo XIX (Aunión, 2009).

Las redes sociales son actualmente utilizadas por personas muy heterogéneas con diferentes edades, niveles de educación, género, condición social, idioma y cultura que participan e incorporan las redes sociales en su vida cotidiana (Mazman y Usluel, 2010) En el Ecuador, cerca de un $98 \%$ de personas que están sobre la franja de edad de los 12 años tiene una cuenta en Facebook (INEC, 2014). El 38,0\% de las personas usó Internet como fuente de información, mientras el 31,5\% lo utilizó como medio de comunicación en general y el 23,2\% como educación y aprendizaje (INEC, 2016). Instagram es una aplicación en línea gratuita que permite a sus usuarios cargar, editar y personalizar fotos y videos. Es la sexta plataforma de redes sociales más utilizada a nivel mundial, con más de mil millones de miembros y más de 500 millones de usuarios diarios, con un $70 \%$ de estos usuarios menores de 35 años (Mansoor, 2021). Esto la convierte en una plataforma prometedora para la recopilación de datos, para aumentar la visibilidad, facilitando un marketing eficaz y un alcance global para todas las empresas, académicos, políticos y redes (Appel et al., 2020). 


\section{Antecedentes}

Los cursos magistrales son un pilar en la instrucción universitaria a pesar de sus limitaciones en relación con el compromiso estudiantil y los resultados de aprendizaje resultantes. En la actualidad se intenta incorporar el uso de redes sociales en la academia para que este espacio sirva como un suplemento voluntario donde los estudiantes puedan discutir acerca del contenido del curso (Cuesta et al., 2015). Es importante de la formación en tecnología para la formación profesional y ejercer su actividad laboral (Salazar et al, 2020).

Son varios los estudios realizados al respecto. Por ejemplo, en el máster universitario de tecnologías de la información y la comunicación (e-learning) de la Universitad Oberta de Catalunya. Se desarrolló un proyecto cuyo objetivo principal fue generar conocimiento sobre las posibilidades de Facebook para el desarrollo de actividades de aprendizaje colaborativo en línea. Se analizó y valoró desde doble enfoque tecnológico y pedagógico. Para ello se creó un grupo privado en la red Facebook y se trasladó a este el proceso de trabajo colaborativo. Se concluyó que las posibilidades pedagógicas son buenas, especialmente si se está dispuesto a mirar con nuevos ojos los conceptos de formación y capacitación y si se enfatiza en el carácter social de la construcción del conocimiento (Cerdà y Planas, 2011).

Los académicos utilizan Instagram para recopilar y difundir datos científicos recientes (Zimba y Gasparyan, 2021). La creciente dependencia de los estudiantes y profesionales de las redes sociales, especialmente durante la pandemia propugnó su uso. Compartir información educativa utilizando infografías y videos cortos en Instagram facilita el aprendizaje (Sinha et al., 2020). Los autores reconocen el potencial de Instagram como herramienta educativa. Las redes sociales aumentan la motivación dentro del aula (Maggiolini, 2013). Mejoran la autoestima y la autoeficacia de los alumnos. Cambia positivamente las percepciones y actitudes. Reduce la ansiedad en los procesos de enseñanza y aprendizaje. Ofrece una oportunidad para resolver problemas en contextos académicos (Aydin, 2012). Fortalecer el espíritu comunitario entre los estudiantes. 
Impulsa el aprendizaje colaborativo, reflexivo. Mejor comunicación entre alumnos y educadores (Schroeder et al., 2010).

Varios contextos de enseñanza y aprendizaje incluyen aprendizaje social, aprendizaje electrónico, aprendizaje ambiental, negocios, arte y educación, química. Las redes sociales puede ser un ambiente educativo valioso. Los educadores deben ser muy sensibles a los efectos negativos de, tales como comportamientos inapropiados, el abuso y el acoso cibernético. Por lo tanto, la ética del uso de redes sociales debe ser considerada y regulada en escalas globales, nacionales y escolares, ya que los resultados muestran que los usuarios necesitan directrices más definidas cuando participan en las redes sociales (Aydin, 2012). Sin duda el consumo de redes sociales es muy alto. Los estudiantes presentan una actitud favorable cuando el docente utiliza las redes sociales como un recurso educativo. Sin embargo, la frecuencia con la que los estudiantes dan un uso académico a las redes es escasa. Esto se deduce a un limitado aprovechamiento por parte de los docentes (Gómez et al., 2012) El valor añadido de las redes sociales es que favorecen un aprendizaje formal e informal, fomentan de la comunicación, tiene facilidad para compartir contenidos y generar foros de discusión y son sencillas de utilizar (Iglesias y González, 2013).

\section{Turismo y las redes sociales}

Mientras la plataforma de Instagram crece y desempeña un papel fundamental, cada vez más central en los medios sociales, es fundamental que los usuarios o los investigadores empiecen a ver que esta plataforma no es sencillamente una herramienta de relación o comunicación. Instagram también funciona como una nueva plataforma de marketing y comunicación de marca donde, debido a las herramientas que la aplicación ofrece, se puede conectar mejor con el público y generar novedosas maneras de conexión para ofrecer material de consumo audiovisual importante, que impacte, enamore y cree la necesidad de adquirir productos (Góngora y Lavilla, 2020).

La red social Instagram se caracteriza por albergar una cantidad alta de publicaciones “inspiracional” cuyo contenido más consumido en la plataforma es el de los viajes con un 47\% (The Cocktail, 2016). Una investigación de Jang et al. (2015) halló que el 91\% 
de los viajeros optan por compartir sus vivencias de viaje por medio de fotos. Existe 14,9 millones de publicaciones con el hasghtag \#viajar y 604 millones de publicaciones con el hashtag \#travel dentro de la plataforma (Instagram, 2021). El uso de Instagram dentro del marketing turístico está aumentando, esta aplicación crea la oportunidad para compartir fotografías, vídeos y otras experiencias turísticas y desafía la imaginación de los socios de viajes para promover destinos turísticos en todo el mundo (Anuar et al., 2021).

Conforme el análisis llevado a cabo por Granberg Linus (2019), indica que los individuos están encontrando nuevos sitios para viajar por medio de Instagram. En el contexto del marketing turístico, las imágenes proporcionan el principal objetivo para que el turista cree una experiencia online del lugar a través de las fotos captadas por los turistas reales, estas imágenes ayudarán a los comercializadores de destinos turísticos por medio de la utilización de la táctica de marketing del boca a boca electrónico (E-WOM) (Kumar, 2020). Las imágenes poseen un monumental efecto en la conducta de compra de los clientes, debido a que les permiten implantar una conexión visual con un producto previo a comprarlo (Tuckman, 2012). Manap (2013) subrayan el papel de las redes sociales en el desarrollo de los destinos turísticos, y Hanan y Putit (2014) recogen la misma expresión una vez que señalan la contribución de las redes sociales como componente de fomento para la promoción de los destinos. La industria del turismo y la zona de la hostelería han desempeñado un papel bastante fundamental en el momento de satisfacer la necesidad de los viajeros de buscar el destino conveniente para sus necesidades de viaje. Internet da un lugar para establecer la toma de elecciones de los turistas ya brinda a los usuarios la posibilidad de participar, desarrollar y actualizar cualquier información.

En su investigación Wegerer y Volo, (2021) concluye que la plataforma Instagram, con sus particulares propiedades es una de las mejores herramientas en línea para generar y recrear la imagen del destino turístico. Recomienda usar fotos y vídeos promocionales representando la belleza, la serenidad y el llamativo del destino. Una revisión de las publicaciones científicas llevadas a cabo sobre redes sociales y turismo muestra que éstas poseen predominación en cada una de las etapas del proceso de viaje, aunque en especial en los periodos previas de inspiración y planeación. Los turistas escogen contenido publicado en las redes sociales, motivados primordialmente por obtener información de 
los viajes y poder inspirarse para hacer la organización de los suyos (Leung et al., 2013). El Ecuador cuenta con suficientes recursos turísticos para ser aprovechados de manera responsable (Vaca y Monge, 2020). Por lo cual, la academia, las organizaciones y los gestores de destinos turísticos deben destinar recursos a conocer estas plataformas y aprovecharlas para la promoción de los destinos turísticos.

\section{Metodología}

De esta forma el presente estudio utiliza las redes sociales para la promoción turística. La red social Place and Travel Ecuador es una página de Instagram cuyo objetivo es dar a conocer el turismo en el Ecuador. Este proyecto nace a raíz de la pandemia del COVID 19 y es usada como una herramienta para compartir contenido turístico creado por los estudiantes.

Para llevar a cabo el análisis se ha tomado como base el modelo PRGS, propuesto por $I A B$ Spain Research para elaborar su estudio sobre la "actividad de las marcas en medios sociales" en los años 2012-2016. Este modelo ha sido utilizado en diversos estudios, se fundamenta en 4 variables: Presencia, Respuesta, Generación y Sugerencia (PRGS). En la tabla 1 se especifica la descripción y la medición de cada una de estas variables.

\section{Tabla 1}

Variables e indicadores del modelo PRGS

\begin{tabular}{|c|c|c|}
\hline Variable & Indicadores & Descripción \\
\hline \multirow{2}{*}{ Presencia } & Número de seguidores & $\begin{array}{c}\text { Establece el número de } \\
\text { seguidores de la red socialdel } \\
\text { hotel }\end{array}$ \\
\hline & $\begin{array}{c}\text { Número de publicaciones y } \\
\text { publicaciones/día }\end{array}$ & $\begin{array}{c}\text { Índica cuantas publicaciones } \\
\text { ha realizado el hotel }\end{array}$ \\
\hline Respuesta & $\begin{array}{l}\text { Número de reacciones / } \\
\text { número de publicaciones }\end{array}$ & $\begin{array}{l}\text { Viene dada por el número de } \\
\text { reacciones (me gusta, me } \\
\text { encanta, etc.) de los usuarios } \\
\text { que los mismos generan ante| } \\
\text { las publicaciones de la marca }\end{array}$ \\
\hline Generación & $\begin{array}{l}\text { Número de comentarios / } \\
\text { número de publicaciones }\end{array}$ & $\begin{array}{l}\text { Viene dada por el número de } \\
\text { comentarios de los usuarios } \\
\text { que los mismos generan ante } \\
\text { las publicaciones de la marca }\end{array}$ \\
\hline Sugerencia & $\begin{array}{l}\text { Número de } \\
\text { recomendaciones / número } \\
\text { de publicaciones }\end{array}$ & $\begin{array}{c}\text { Viene dada por el número de } \\
\text { comparticiones o } \\
\text { recomendaciones de los } \\
\text { usuarios que los mismos } \\
\text { generan ante las publicaciones } \\
\text { de la marca }\end{array}$ \\
\hline
\end{tabular}


Para una mejor interpretación de los resultados las variables de "respuesta", "generación" y "sugerencia" se han unido en una sola variable denominada "engagement", que corresponde a la suma de las tres anteriores. La formulación y el cálculo del "engagement" ha sido tratada en varios estudios.

\section{Resultados}

En este apartado se determina los resultados obtenidos en el análisis de la intervención y la actividad en la página de Instagram Place and Travel Ecuador durante el 19 de mayo del 2020 hasta el 10 de diciembre del 2021 teniendo en cuenta las variables ya comentadas de presencia y engagement.

Presencia. En el estudio para comprobar la presencia en la página Place and Travel se analizó el número de publicaciones y el número de seguidores. En la figura 1 podemos observar que han existido 268 publicaciones relacionadas a fotografías 70 reels y 37 videos. Todas las publicaciones son autoría de los estudiantes y se han subido a la página de forma periódica según un cronograma de publicación.

\section{Figura 2}

Contenido publicado

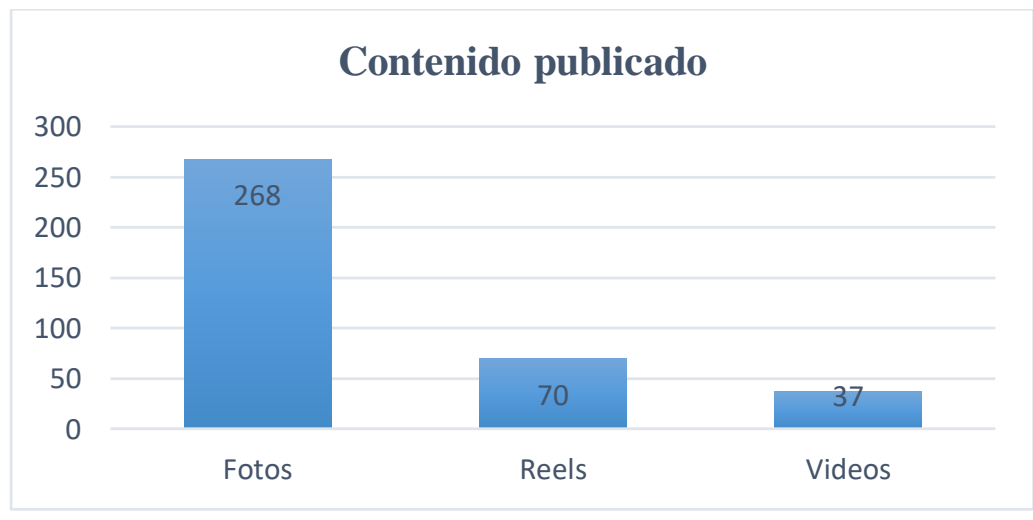

Fuente: Facebook Business 
En cuanto al número de seguidores la red cuenta con 1,800 seguidores a la fecha del 10 de diciembre del 2021. En resumen, se muestra las fluctuaciones que se han ido generando en el alcance a medida que se ha ido publicando los distintos tipos de contenido, es así como se puede mencionar al ver la figura 2 que existen 4 periodos en los que se ha tenido activa dicha red social y se comprende por periodos que van acorde a la administración de los diferentes cursos a cargo de las publicaciones de la página.

\section{Figura 3}

Análisis general del alcance en Instagram

Alcance de Instagram (9)

\subsection{5}

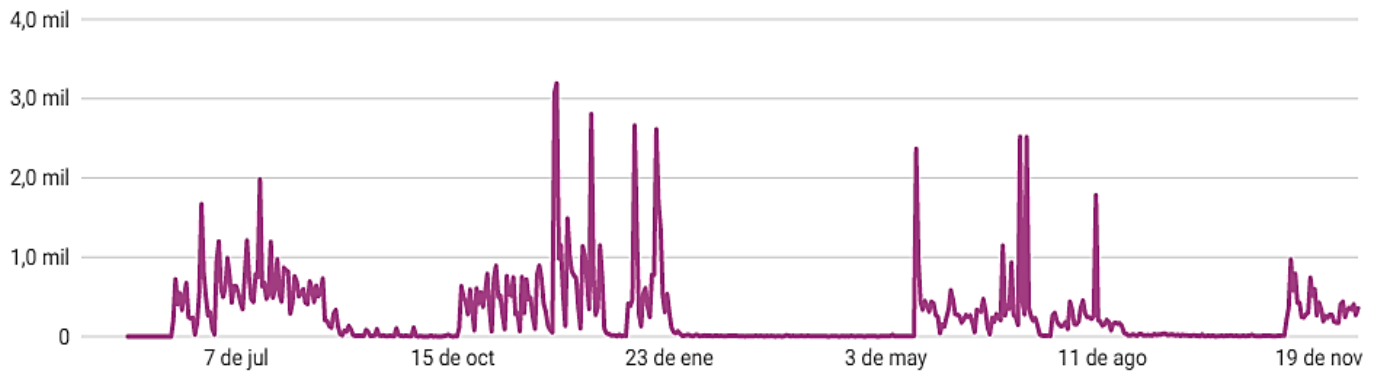

Fuente: Facebook Business

Como se observó en la figura 2, los cuatro periodos que van desde la creación de la página se mantiene fluctuaciones e intervalos en los que se puede medir la mayor o menor cantidad de alcance que obtuvo la página en el transcurso del tiempo. Cabe recalcar que como observación se puede decir que existen varios factores que influyen en dichos resultados de los intervalos y sus fluctuaciones como la cantidad y frecuencia del contenido publicado, la hora de publicación, la cantidad de seguidores, de igual forma los distintos algoritmos en el cual se manejan las redes sociales y el objetivo que va dirigido dicho contenido. 


\section{Figura 4}

Datos demográficos de Instagram

Seguidores de Instagram (6)

1,8 mil

Edad y sexo 0
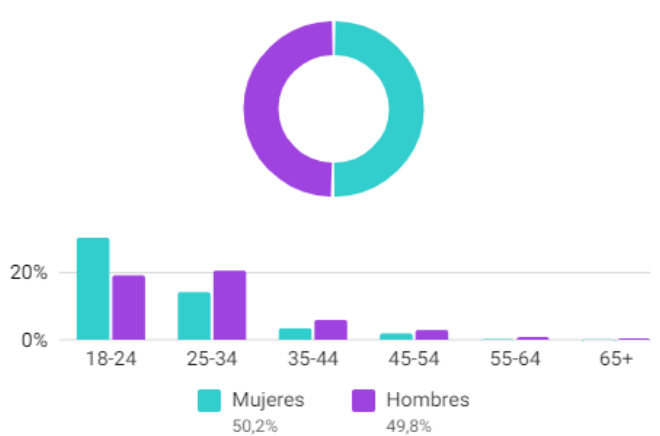

Principales ciudades

Ambato, Ecuador

Quito, Ecuador

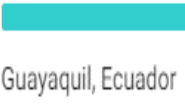

$4.5 \%$

Pillaro, Ecuador

$2.8 \%$

Latacunga, Ecuador

$2.5 \%$
Principales países

Ecuador

$77.3 \%$

Estados Unidos

$1.8 \%$

Colombia

| $0.7 \%$

España

| $0.6 \%$

México

| $0.6 \%$

Fuente: Facebook Business

En la figura 3 se da a conocer un resumen de los datos demográficos alcanzados en Instagram, dando como resultado que el mayor objetivo de la página tiende a ser personas de sexo femenino entre una edad de 18 a 24 años seguido por personas del sexo masculino entre una edad entre los 25 y 34 años. Él lo que compete a los principales países se observa que la mayor parte de usuarios alcanzados son de nacionalidad ecuatoriana con residencia en la ciudad de Ambato seguidos por Quito, Guayaquil, Píllaro y Latacunga, posterior a 
ello y en menor cantidad existen usuarios alcanzados de países como Estados Unidos seguido de Colombia, España y México.

Una vez comprendido el contexto que se ha ido formando durante el proceso de publicación en la página de Place and Travel Ecuador podemos mencionar que para esta segunda parte se dará a conocer datos más específicos sobre las variables de engagement.

Engagenment. Una vez analizada la presencia en la página de Instagram de Place and Travel Ecuador para la promoción turística se analiza la variable engagenment entendida como el número de interacciones realizadas por los usuarios que se produce por cada publicación.

\section{Figura 4}

\section{Alcance de las publicaciones en Instagram}

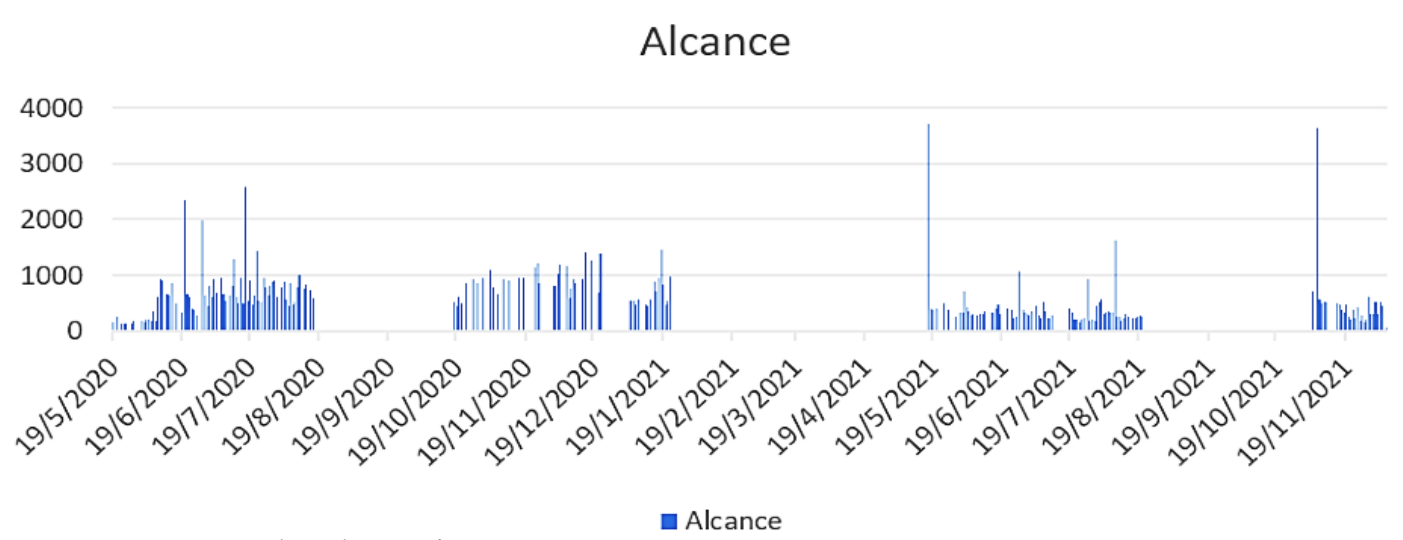

Fuente: Facebook Business

Como se puede observar en la figura 4 obtenemos que en los 4 periodos han existido diferentes tipos de intervalos en los que podemos destacar a las publicaciones con mayor y menor número de alcance, siendo 3702 el mayor número de usuarios alcanzados perteneciente a un reel publicado el 17 de mayo del 2021, del periodo número tres. Un tipo de video corto de 50 segundos en el que se puede agregar varias ediciones y animaciones para hacer más atractiva la visualización de dicho clip. De igual forma los reels en el algoritmo de Instagram ayudan a captar la atención de la audiencia y de los posibles seguidores. Se puede intuir que, por factores como el algoritmo de Instagram, el plan de marketing aplicado o la interacción misma de la audiencia actual ayudó a que dicha publicación tenga el mayor alcance posible. 


\section{Figura 5}

Número de me gustas de las publicaciones en Instagram

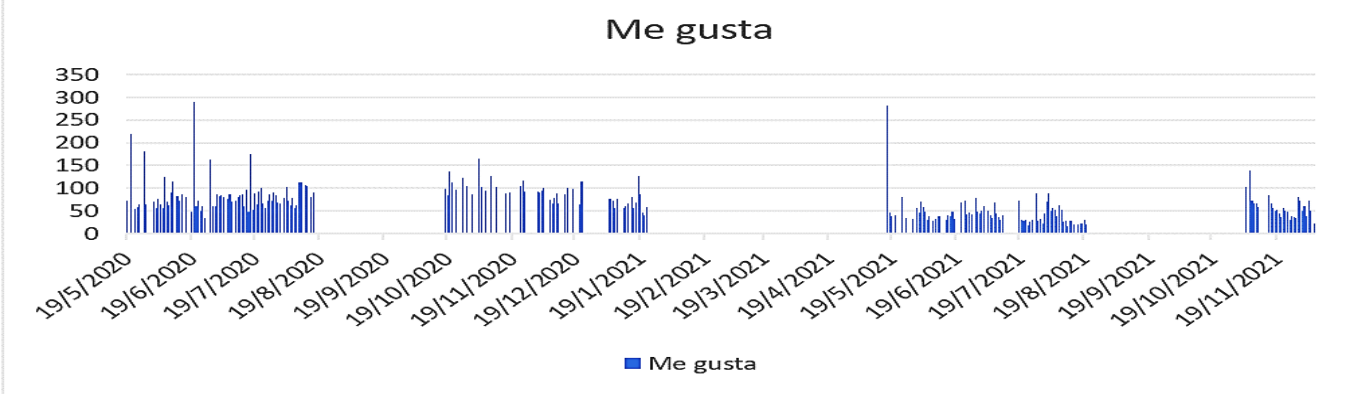

Fuente: Facebook Business

En lo que compete a las internaciones o número de me gustas se puede observar el gráfico N. 5 que se destaca a las publicaciones con mayor y menor número de interacciones, siendo 290 el mayor número de me gustas recibidos en un solo post perteneciente a la publicación del 20 de junio del 2020 del periodo número uno, cuyo contenido es una fotografía. Cómo se mencionó anteriormente el número de me gustas puede ser influenciado por factores como la interacción de la audiencia, la hora de publicación, el plan de marketing aplicado y de igual forma en este caso la fotografía como tal. Este post cumple con los parámetros que posee una buena fotografía en factores como una buena composición, buen

\section{Figura 6}

Número de comentarios de las publicaciones en Instagram

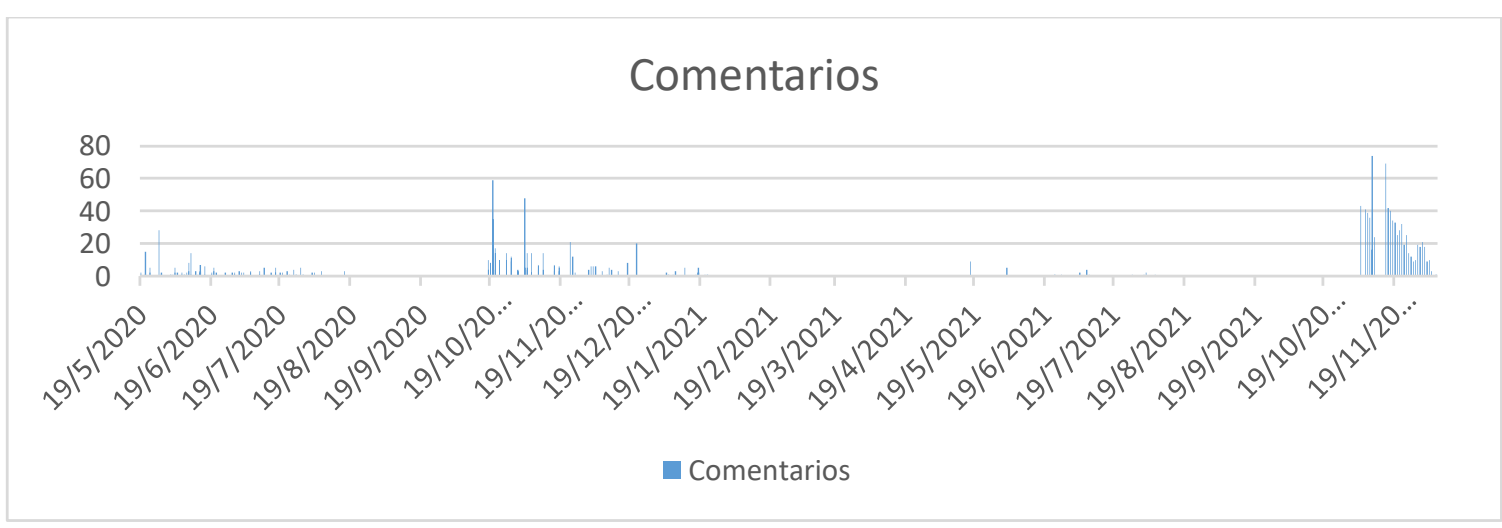

Fuente: Facebook Business 
En referencia a los comentarios como se puede observar en la figura 6 las publicaciones con mayor y menor número de comentarios es el reel del 09 de noviembre del 2021 con 74 comentarios. No se puede dejar atrás la interacción de la audiencia con comentarios positivos relacionados con la temática o el contenido emitido en dicho reels. A su vez se puede mencionar que en el periodo 4 se observa el mayor número de comentarios en las publicaciones debido a los distintos tipos de contenido como videos, reels y fotografías. Además, se exponen contenidos e información de valor y más interactivos. La audiencia aprueba e interactúan en mayor medida los comentarios que posee la publicación.

\section{Figura 7}

Número de veces compartidas de las publicaciones en Instagram

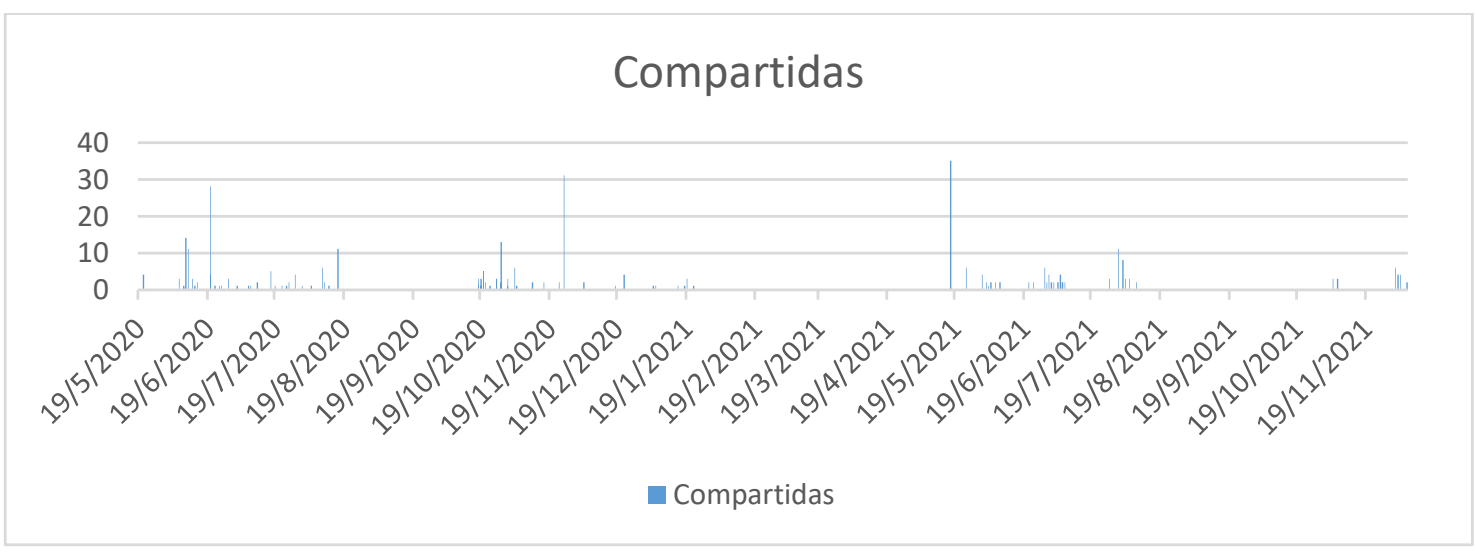

Fuente: Facebook Business

Por último, en la figura 7 se observa las veces que más ha sido compartida una publicación siendo 35 el mayor número en la publicación del 17 de mayo del 2021 del periodo tres siendo esta publicación un reels. Esta publicación también fue la que mayor comentario tuvo. Por lo tanto, se puede intuir que el número de veces compartidas influye en el número de alcance. No obstante, en el mismo contexto se puede mencionar que en el periodo uno existe un mayor número de veces de publicaciones compartidas. Mediante estos datos y la observación de las publicaciones se puede analizar que las más compartidas son las publicaciones que se encuentran con una mejor edición, animación, 
revelado, encuadre y el mensaje se transmite de mejor manera ya sea de cualquier tipo de contenido.

\section{Conclusiones}

- Como conclusión se puede mencionar que se ha realizado un completo estudio sobre las estrategias de promoción en la red social Instagram. Las publicaciones han sido constantes durante los cuatro períodos académicos desde el 19 de mayo del 2019 que inició la modalidad de educación virtual hasta el 10 de diciembre del 2021. Se han realizado 289 publicaciones fotográficas, 70 reels y 37 videos lo que denota un trabajo constante de los alumnos. Las publicaciones varían en su alcance, interacción, número de comentario y veces compartidas ya sea por influencia de distintos factores como la engagement del público de cada red social, el algoritmo base de la plataforma, horarios de publicación y contenido publicado.

- En cuanto a la promoción turística que se genera en Instagram se analizó el alcance. Es así como las fotografías, videos, y reels han llegado a futuros turistas nacionales como internacionales. El 77\% son turistas nacionales procedentes de Ambato, Quito, Guayaquil, Pillarlo, Latacunga. El 1,8\% son de Estados Unidos y en un menor porcentaje de Colombia, España y México. Se resalta en alcance internacional que tiene esta red social, hecho que se debe tomar en cuenta para la promoción turística de los destinos.

- El crecimiento continúo en la interacción de los usuarios es algo que se debe aprovechar por parte de las entidades públicas y la academia. Realizar estrategias específicas para atraer a más usuarios y generar un contenido idóneo. Especialmente si se está dispuesto a mirar con nuevos ojos los conceptos de formación y capacitación y si se enfatiza en el carácter social de la construcción del conocimiento. En lo que compete al análisis de las fotografías se pude observar que las fotos con mayor alcance y número de me gustas, son las fotografías que presentan una mayor aprobación y cumplimiento de los distintos parámetros salvo algunas fotos que necesitan mejorar en su edición. Mientras que las fotografías con menor número de alcance y número de me gustas son las fotografías que presentan una poca aprobación y déficit en los distintos parámetros de edición. 
- Esta información debe ser aprovechada tanto para la promoción de destinos turísticos como para utilizar herramientas tecnológicas en varios contextos de enseñanza y aprendizaje que incluyan un aprendizaje social, un aprendizaje electrónico, aprendizaje ambiental, que permitan la participación de estudiantes en la generación de contenidos turísticos.

\section{Referencias bibliográficas}

Abuín, N. (2009). Las redes sociales como herramienta educativa en el ámbito universitario. Relada (Revista Electrónica de ADA) 3 (3): 199205. http://polired.upm.es/index.php/relada/article/viewFile/78/78.

Anuar, F., Zaid, A., Zabidi, I. \& Kamal, S. (2021). Young Tourists' Trust in Instagram Travel Influencers and their Intention to Visit Travel Destinations. International Journal of Academic Research in Business and Social Sciences, 11(16). https://doi.org/10.6007/ijarbss/v11-i16/11236

Appel, G., Grewal, L., Hadi, R. \& Stephen, A. T. (2019). The future of social media in marketing. Journal of the Academy of Marketing Science, 48(1), 79-95. https://doi.org/10.1007/s11747-019-00695-1

Aunión, J. (2009). La era del profesor desorientado. El país.

https://webs.um.es/rhervas/miwiki/lib/exe/fetch.php?media=la_era_del_profesor _desorientado_elpais.com.pdf

Aydin, S. (2012). A review of research on Facebook as an educational environment. Educational Technology Research and Development, 60(6), 10931106. https://doi.org/10.1007/s11423-012-9260-7

Caerols, R., Tapia, A. \& Carretero, A. (2013). Instagram, la imagen como soporte de discurso comunicativo participado. Vivat Academia, O(124), 68. https://doi.org/10.15178/va.2013.124.68-78

Cerdà, F. \& Planas, N. (2011). Facebook's Potential for Collaborative eLearning. RUSC. Universities and Knowledge Society Journal, 8(2), 31. https://doi.org/10.7238/rusc.v8i2.963

Cuesta, M., Eklund, M., Rydin, I. \& Witt, A. K. (2015). Using Facebook as a colearning community in higher education. Learning, Media and Technology, 41(1), 55-72. https://doi.org/10.1080/17439884.2015.1064952

Espuny Vidal, C., González Martínez, J., Lleixà Fortuño, M. \& Gisbert Cervera, M. (2011). University Students' Attitudes Towards and Expectations of the Educational Use of Social Networks. RUSC. Universities and Knowledge Society Journal, 8(1). https://doi.org/10.7238/rusc.v8i1.839 
Fernández, T. (2014). Aprendizaje colaborativo y uso de las redes sociales en educación primaria. Didáctica. Lengua y Literatura, 25(0). https://doi.org/10.5209/rev_dida.2013.v25.42240

Figuereo-Benítez, J. C., González, F. \& Machin, J. D. (2021). Instagram como objeto de estudio en investigaciones recientes. Una revisión de literatura con enfoque en revistas científicas. Ámbitos. Revista Internacional de Comunicación, 53, 9-23. https://doi.org/10.12795/ambitos.2021.i53.01

Garrigós, I., Mazón, J.-N., Saquete, E., Puchol, M. \& Moreda, P. (2010). La Influencia de las redes sociales en el aprendizaje colaborativo. XVI Jornadas de Enseñanza Universitaria de La Informática, 531-534.

Gómez, M., Roses, S. \& Farias, P. (2012). The Academic Use of Social Networks among University Students. Comunicar, 19(38), 131-138. https://doi.org/10.3916/c38-2012-03-04

Góngora, G. \& Lavilla, D. (2020). La importancia de la construcción de marca en Instagram para las empresas periodísticas. Estudios multidisciplinarios en comunicación audiovisual, interactividad y marca en la red, 12938. https://hdl.handle.net/11441/95647.

Granberg, L. (2019). "The role of instagram in choosing a travel destination". Studentthesis, Luleå tekniska universitet, Institutionen för ekonomi, teknik och samhälle. http://urn.kb.se/resolve?urn=urn:nbn:se:Itu:diva-76377.

Hanan, H. \& Putit, N. (2013). Express marketing of tourism destinations using Instagram insocial media networking. Hospitality and Tourism, 471-474. https://doi.org/10.1201/b16064-93

Iglesias, M. \& González, C. (2013). El uso de Facebook como herramienta para la interacción en el proceso de enseñanza-aprendizaje. XI Jornadas de Redes de Investigación En Docencia Universitaria 2013, 12(Language), 1-10. Retrieved from http://m.web.ua.es/es/ice/jornadas-redes/documentos/2013-comunicacionesorales/335221.pdf

INEC. (2014). Compendio de Resultados Encuesta de Vida, sexta ronda 2015. Inec, 197. https://doi.org/10.1007/s13398-014-0173-7.2

INEC. (2016). Tecnologías de la Información y Comunicaciones (TIC’S 2016.

Instagram. (2021). Instagram [\#viajar]. https://www.instagram.com/explore/tags/viajar/

Jang, J. Y., Han, K., Shih, P. C. \& Lee, D. (2015). Generation Like. Proceedings of the 33rd Annual ACM Conference on Human Factors in Computing Systems. https://doi.org/10.1145/2702123.2702555 
Kumar, V. (2020). Yonder Glimpses: Role of Instagram in creating tourism branding. Mukt Shabd Journal, IX(IV), 2500-2512. http://shabdbooks.com/gallery/254april2020.pdf

Leung, D., Law, R., van Hoof, H. \& Buhalis, D. (2013). Social Media in Tourism and Hospitality: A Literature Review. Journal of Travel \& Tourism Marketing, 30(1-2), 3-22. https://doi.org/10.1080/10548408.2013.750919

Maggiolini, L. (2013). Estrategias de motivación en una era digital: Teléfonos móviles y Facebook en el aula Motivational Strategies in a Digital Age: Phones and Facebook in a classroom, (24), 83-98.

Manap, K. (2013). The role of User generated Content (UGC) insocial media for Tourism Sector. The 2013 WEI International Academic Conference Proceedings, Istanbul-Turkey

Mazman, S. G. \& Usluel, Y. K. (2010). Modeling educational usage of Facebook. Computers \& Education, 55(2), 444-453. https://doi.org/10.1016/j.compedu.2010.02.008

Mansoor, I. (2021, 12 November). Instagram Revenue and Usage Statistics (2021). Business of Apps. https://www.businessofapps.com/data/instagram-statistics/

Moreno, M. (2018). La enciclopedia del community manager. Barcelona:

Deusto. https://www.planetadelibros.com/libros_contenido_extra/38/37723_La_e nciclopedia_del_Community_Manager.pdf.

Newberry, C. (2021, 3 febrero). 44 Instagram Statistics That Matter to Marketers in 2021. Social Media Marketing \& Management Dashboard. https://blog.hootsuite.com/instagram-statistics/

Salazar. J., Moge, J. \& Armas, S. (2020). Análisis de los profesionales de turismo y hotelería de la Universidad Técnica de Ambato en el uso de las tecnologías de la información. Kalpana- Revista de Investigación, 19, 7-20.

Schroeder, A., Minocha, S. \& Schneider, C. (2010). The strengths, weaknesses, opportunities and threats of using social software in higher and further education teaching and learning. Journal of Computer Assisted Learning, 26(3), 159-174. https://doi.org/10.1111/j.1365-2729.2010.00347.x

Sinha, M., Agarwal, V. \& Gupta, L. (2020). Human touch in digital education-a solution. Clinical Rheumatology, 39(12), 3897-3898. https://doi.org/10.1007/s10067-020-05448-y

The Cocktail Analysis. (2016, 18 diciembre). VIII Ola del Observatorio de Redes Sociales. InformeTICfacil.com. https://www.informeticplus.com/viii-ola-delobservatorio-de-redes-sociales-the-cocktail-analysis-y-arena 
Tuckman, B. (2012, 6 junio). How Photography and Online Video Influence Customer Purchasing Behavior. MarketingProfs. https://www.marketingprofs.com/articles/2012/8088/how-photography-andonline-video-influence-customer-purchasing-behavior

Vaca Vaca, C. I. \& Monge Martínez, J. G. (2020). El Ecoturismo y los factores de influencia en la experiencia turística durante excursiones en áreas naturales: Laguna del Quilotoa. ConcienciaDigital, 3(3.2), 47-59. https://doi.org/10.33262/concienciadigital.v3i3.2.1407

Wegerer, P. \& Volo, S. (2021). Visual Content Analysis of Instagram posts: The case of an Alpine destination. Travel and Tourism Research Association: Advancing Tourism Research Globally. 50. https://scholarworks.umass.edu/ttra/2021/research_papers/50

Zimba, O. \& Gasparyan, A. Y. (2021). Social media platforms: a primer for researchers. Reumatologia/Rheumatology, 59(2), 68-72. https://doi.org/10.5114/reum.2021.102707

El artículo que se publica es de exclusiva responsabilidad de los autores y no necesariamente reflejan el pensamiento de la Revista Conciencia Digital.

\section{Liencia}

El artículo queda en propiedad de la revista y, por tanto, su publicación parcial y/o total en otro medio tiene que ser autorizado por el director de la Revista Conciencia Digital.
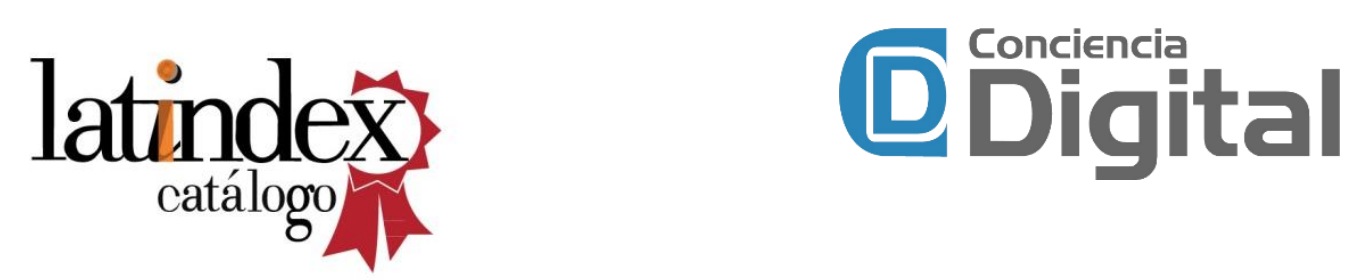

Indexaciones

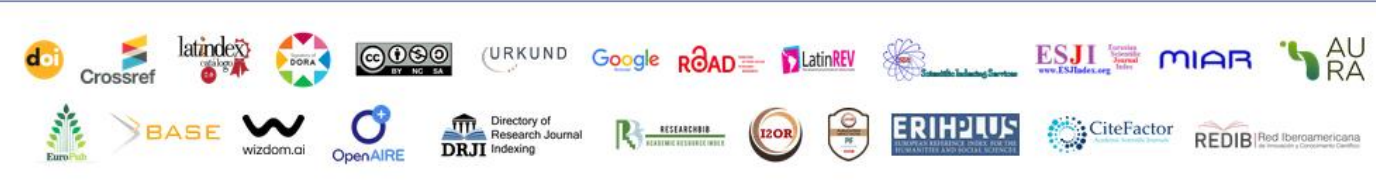

